Conclusion Across undergraduate training students have exposure to a broad range of experiences in which care of the dying is demonstrated. However, students may not always recognise when they are witnessing care of the dying. Different settings provide different experiences but consolidate common principles. These experiences have considerable impact and students expressed a need for further support. Our low response rate may affect our findings.

\title{
P 061 HOW MEDICAL STUDENTS LEARN TO CARE FOR THE DYING: RESULTS FROM A QUESTIONNAIRE STUDY
}

Clare Rayment, ${ }^{1}$ Rebecca O'Rourke ${ }^{2} .{ }^{1}$ Marie Curie, Bradford, UK; ${ }^{2}$ University of Leeds

10.1136/bmjspcare-2014-000654.102

Aims To explore the exposure medical students have across clinical placements in learning to care for dying patients, with a particular focus on whether there are perceived differences relating to learning in different settings.

Methods All 4th and 5th year medical students at one UK medical school were invited to complete a short online questionnaire. Data was analysed with descriptive statistics and thematic analysis.

Results Response rate was 4\%. 22 students responded, 15 from fourth and 7 from fifth year. 18 perceived they had been involved in the care of a dying patient and 15 had completed a module including palliative care during medical school. Students described many varied experiences across a broad range of specialities of caring for patients dying but many also had prior experience outside their medical training. Students described learning through role modelling from both perceived positive and negative encounters. Students identified similarities in underlying principles across care settings but described differences related to staff attitudes and priorities. The Liverpool Care Pathway was often mentioned as a framework for understanding how to care for the dying. Many students described the considerable impact of being involved, even on the periphery, of caring for a dying patient. Despite 4 students stating they had not been involved in the care of a dying patient, in subsequent questions they described these experiences; they were all final year medical students. 